\title{
ESTIMATIVA DO TAMANHO MÍNIMO DE REBANHO SUÍNO PARA A IMPLEMENTAÇÃO DE SISTEMA DE GERAÇÃO DE ENERGIA ELÉTRICA DE 35 KWH, 150 KWH, 275 KWH E 590 KWH, USANDO BIOGÁS COMO COMBUSTÍVEL PARA GRUPOS GERADORES
}

\author{
Diego Augusto Gonzaga ${ }^{1}$, Rúben Christian Barbosa²
}

\begin{abstract}
RESUMO - O Brasil é um grande produtor de suínos para exportação e para o abastecimento do mercado interno. Porém essa atividade possui um grande impacto ambiental devido à grande geração de resíduos. Os suínos poluem em média aproximadamente 4,5 vezes mais que um ser humano. Uma das soluções é a implementação de biodigestores para o tratamento desses resíduos, o que possibilita a geração de biogás para ser utilizado como combustível para grupos geradores de energia. Esse trabalho buscou elaborar um modelo matemático para estimar qual é o número ótimo de animais pertencentes a um rebanho de uma granja suína para a implementação de sistemas de geração de energia elétrica. Foram levantados dados de necessidade energética, enquadramentos tarifários, valores de investimento para a implantação e capacidade de produção dos sistemas. Após realizada a modelagem e utilizando o software Lingo 14.0 Versão Demo foram calculados os valores ótimos para o sistema de geração de energia. Os resultados obtidos pelas simulações quando comparados a casos reais se mostram bastante razoáveis, validando a modelagem. Os dados de tamanho de rebanho obtidos se mostraram razoáveis e factíveis se comparados a empreendimentos reais na região do vale do Piranga.
\end{abstract}

Palavras chave: biogás, energia elétrica, modelagem, suíno.

\section{ESTIMATE OF THE MINIMUM SIZE OF A SWINE HERD FOR THE IMPLEMENTATION OF ELECTRIC POWER GENERATION SYSTEM OF 35 KWH, $150 \mathrm{KWH}, 275 \mathrm{KWH}$ AND $590 \mathrm{KWH}$, USING BIOGAS AS FUEL FOR GENERATORS}

\begin{abstract}
Brazil is a big pork producer either to export or to supply the domestic market. However, the productive activity generates a great environmental impact due its large amount of waste. Each pig pollutes averagely about 4.5 times more than a human being. One of the solutions is to implement biodigestors to the waste treatment which can provide bio gas to be utilized as fuel for energy generators. The research has tried to elaborate a mathematical model to estimate which is the optimal number of animals in the herd of a swine farm to implement an electrical energy generator system. There were a data collection such as energy needs, tariff frameworks, investment costs to an implementation and the production capacity of the systems. After performing the model taking the software Lingo 14.0 Demo Version, the optimum values were calculated for the energy generation system. Once taken the simulation data compared to the actual data the results obtained were very reasonable, validating the model. The herd size data have shown they are reasonable and operable whether comparing to the real target implementation of The Vale do Piranga enterprise.
\end{abstract}

Keywords: biogas, eletric power, modeling, swine.

\footnotetext{
${ }^{1}$ Engenheiro de Controle e Automação, UFV, (31)3899-2732, gonzagaaugusto@ gmail.com

${ }^{2}$ Engenheiro de Controle e Automação, UFV, (31)3899-2732, rubenchristianbarbosa@ hotmail.com
} 


\section{INTRODUÇÃO}

O Brasil é quarto maior produtor e exportador de carne suína no mundo, com $3 \%$ da produção, $11 \%$ das exportações e crescente inserção internacional. $\mathrm{O}$ desempenho brasileiro na década passada foi positivo, passando de $4 \%$ para $11 \%$ das exportações mundiais, com aproximadamente 530 mil toneladas exportadas em 2008. O mercado interno com mais de 191 milhões de habitantes e o seu dinamismo têm garantido uma sólida base de expansão da cadeia produtiva, sobretudo nos anos de retração da demanda (Miele \& Machado, 2010).

A cada dia a produção de carne suína é um fator mais expressivo na balança econômica brasileira. De acordo com Secretaria de Estado da Agricultura e do Abastecimento - Departamento de Economia Rural (2013), o rebanho brasileiro no ano de 2011 era de 38,9 milhões de cabeças, e foram exportadas 576,8 mil toneladas de carne.

Porém a produção de suínos exige grande atenção por parte de órgão ambientais e dos empreendedores do ramo. A produção de suínos é uma atividade que gera grande quantidade de resíduos devido ao grande volume de produção de dejetos pelos animais. Os suínos poluem, em média, aproximadamente 4,5 vezes mais que um ser humano e, na maioria dos casos, os dejetos produzidos são descartados in natura diretamente nos corpos de água receptores (Godoy Júnior et al., s.d.).

Uma solução para o controle dos impactos ambientais causados por esse processo produtivo é a implementação de biodigestores. Esses dispositivos são câmaras que realizam a fermentação anaeróbia da matéria orgânica produzindo biogás e biofertilizante. Processo da digestão anaeróbia consiste na transformação de compostos orgânicos complexos em substâncias mais simples, como metano e dióxido de carbono, através da ação combinada de diferentes microrganismos que atuam na ausência de oxigênio. O biodigestor pode ser construído de pedra ou tijolo e a campânula de ferro, fibra de vidro ou PVC. No Brasil o modelo contínuo foi o mais difundido pela sua simplicidade e funcionalidade (Embrapa, 2002).

Ainda de acordo com dados do Embrapa (2002), no meio rural pode-se atender quase que totalmente às necessidades energéticas básicas, tais como: cozimento, iluminação e geração de energia elétrica para diversos fins. Biofertilizante é o efluente resultante da fermentação anaeróbia da matéria orgânica, na ausência de oxigênio, por um determinado período de tempo. Pode ser utilizado como adubo do solo tanto puro quanto na formação de compostagens.

A produção de biogás a partir de um determinado volume do biodigestor pode variar em relação a vários fatores como temperatura ambiente, nível de diluição do material, entre outros fatores. Outro fator que influencia na produção é o modelo de biodigestor implementado. Essas variações fazem com que a determinação exata de produção de biogás por um biodigestor seja difícil. Porém existem estimativas que fornecem um valor médio de produção de biogás.

Esse trabalho buscou elaborar um modelo matemático para estimar qual é o número ótimo de animais pertencentes a um rebanho de uma granja suína para a implementação de sistemas de geração de energia elétrica.

\section{MATERIAL E MÉTODOS}

O gás produzido pelo biodigestor é utilizado em motores a combustão interna de grupos-geradores para a produção de energia elétrica. O potencial de produção de energia é proporcional a produção de gás que por sua vez é proporcional ao número de animais da granja.

Analisando de forma global as instalações, de toda carga instalada em uma granja, normalmente, $76 \%$ do total se encontra em locais mais diretamente ligados ao processo produtivo e $24 \%$ no escritório e residências (Ferrarez et al., 2010).

Coldebella et al. (2008), em seu estudo sobre a viabilidade da geração de energia elétrica através de um motor gerador, utilizando biogás da suinocultura, conclui que o tempo de retorno do investimento em geração de energia própria tornasse atrativo com o uso intensificado do sistema nos empreendimentos. Porém, ele considera necessário que as concessionárias adquiram o excedente de energia produzida.

\section{Produção de esterco e gás}

A quantidade total de esterco produzida por um suíno varia de acordo com o seu desenvolvimento ponderal, como mostra a Tabela 1, mas apresenta valores decrescentes de $8,5 \%$ a $4,9 \%$ em relação a seu peso vivo por dia para a faixa de 15 a $100 \mathrm{~kg}$. Cada suíno adulto produz em média de $0,21 \mathrm{~m}^{3}$ a $0,24 \mathrm{~m}^{3}$ de dejetos por mês (Embrapa, 2002). 
A nível de projeto, como mostrado na Tabela 2, foi desenvolvida uma recomendação prática, onde a quantidade de dejetos é estimada de acordo com o sistema produtivo utilizado pelo produtor e com o grau de desperdício da água na granja (Embrapa, 2002).

De acordo com Ferrarez et al. (2010), o cálculo da produção total de biogás de um granja pode ser feito por meio da Equação 1, considerando a produção diária de dejetos de cada suíno igual a $2,5 \mathrm{~kg}$ e a produção de $1 \mathrm{~m}^{3}$ de biogás a partir de $12 \mathrm{~kg}$ de dejetos de suíno.

\section{Equação 1 - Produção de biogás}

$$
\mathrm{Pb}=365 \mathrm{n}_{\mathrm{s}} \cdot \mathrm{d}_{\mathrm{s}} \cdot \mathrm{V}_{\mathrm{bs}}
$$

Fonte: Ferrarez et al. (2010).

Em que:

$\mathrm{Pb}=$ Produção anual de biogás $\left(\mathrm{m}^{3}\right.$ ano $\left.^{-1}\right)$;

ns = número total de suínos da granja (adimensional);

ds = Produção diária de dejetos por suíno $(\mathrm{kg})$; e

Vbs $=$ Volume de biogás por quilo de dejeto de suíno $\left(\mathrm{m}^{3} \mathrm{~kg}^{-1}\right)$

(Ferrarez et al., 2010).

\section{Produção de energia}

O cálculo da energia elétrica produzida a partir do biogás pode ser feito considerando a conversão realizada por meio de Motor Ciclo Otto e Microturbina. Considerando-se como em Ferrarez et al. (2010), concentração de metano $\left(\mathrm{CH}_{4}\right)$ de $62 \%$ e o poder calorífico inferior do biogás igual a $22600 \mathrm{~kJ} \mathrm{~m}^{-3}$, pode-se aplicar a relação apresentada pela Equação 2.

\section{Equação 2 - Energia elétrica gerada por motor de ciclo Otto}

$$
\mathrm{E}_{\text {mco }=} \text { PCI. } \mathrm{B}_{\text {restante. }} \eta_{\text {motor }} \Delta_{\text {top. } 0,001}
$$

Fonte: Ferrarez et al. (2010).

Em que:

$\mathrm{E}_{\text {mco }}=$ energia elétrica gerada por motor ciclo Otto (MWh);

$\mathrm{PCI}=$ poder calorífico inferior do biogás $\left(\mathrm{kJ} \mathrm{m}^{-3}\right)$;

$\mathrm{B}_{\text {restante }}=$ biogás restante $\left(\mathrm{m}^{-3} \mathrm{~s}^{-1}\right)$;

$\eta_{\text {motor }}=$ rendimento do motor ciclo Otto (adimensional); $\mathrm{e}$

$\Delta_{\text {top }}=$ tempo de operação do sistema $\left(\mathrm{h} \mathrm{ano}^{-1}\right)$.

A energia gerada deve ser aplicada inicialmente nos pontos de demanda do próprio empreendimento. Além dos benefícios de independência energética, deve-se levar em conta a economia de recursos proporcionada pela geração própria. Nos cálculos do custo da produção de leitões (UPL) e de suínos em ciclo completo e nos cálculos do custo da produção da terminação a energia elétrica é considerada um custo variável no processo de produção (EMBRAPA, 2006).

Tabela 1 - Produção de esterco por suínos

\begin{tabular}{lccc}
\hline Categoria & Esterco $\left(\mathrm{kg} \mathrm{dia}^{-1}\right)$ & Esterco + urina $\left(\mathrm{kg} \mathrm{dia}^{-1}\right)$ & Dejetos líquidos $\left(\right.$ litros dia $\left.{ }^{-1}\right)$ \\
\hline Suínos $(25-100 \mathrm{~kg})$ & 2,30 & 4,90 & 7,00 \\
Porca gestação & 3,60 & 11,00 & 16,00 \\
Porca lactação + leitões & 6,40 & 18,00 & 27,00 \\
Cachaço & 3,00 & 6,00 & 9,00 \\
Leitões na creche & 0,35 & 0,95 & 1,40 \\
\hline
\end{tabular}

Fonte: Embrapa (2002).

Tabela 2 - Produção de esterco de acordo com o sistema produtivo

\begin{tabular}{lccc}
\hline Tipo de granja & \multicolumn{2}{c}{ Nível de Diluição } & Muita \\
\hline & Pouca & Média & 200 \\
Ciclo Completo $\left(\right.$ I Matriz $\left.^{-1}\right)$ & 100 & 150 & 120 \\
UPL $\left(\right.$ I Matriz $\left.^{-1}\right)$ & 60 & 90 & 15 \\
UT $\left(\right.$ I Matriz $^{-1}$ ) & 7,5 & 11,2 & \\
\hline
\end{tabular}

Fonte: Embrapa (2002). 


\section{Consumo de energia e análise de custos}

O consumo específico nas granjas é estimado por Talamini et al. (2006), em $150 \mathrm{kWh}$ por matriz alojada ao custo de 27 centavos e custo anual de $\mathrm{R} \$ 3.402,00$. Os gastos com energia e combustível foram estimados em $0,5 \%$ dos gastos com mão-de-obra, rações e conservação e reparos, perfazendo um total de $\mathrm{R} \$ 336,10$ por lote, $\mathrm{R} \$ 0,67$ por animal, ou $\mathrm{R} \$ 0,006$ por $\mathrm{kg}$.

De acordo com Noronha \& Gimenes (2008), o gasto específico com de energia em uma granja é de $R \$ 2,16$ e pelos dados levantados por Ferrarez et al. (2010), pode-se chegar a relação de 3,560872 mensalmente.

Para um empreendimento como uma granja de suínos, atualmente o melhor enquadramento tarifário é o de Tarifa Azul A4. Essa tarifação, de acordo com a resolução $n^{\circ} 1507$ - Anexo I de 05/04/2013, vigorando a partir de 08/04/2013, estabelece tarifas de demanda e consumo, dentro e fora do período de ponta, como mostra a Tabela 3.

\section{Método de otimização}

A otimização é o processo de encontrar a melhor solução dentro de um conjunto de soluções para um determinado problema. No mundo real, muitos processos podem se beneficiar de uma alocação otimizada de recursos. Esses recursos, que podem incluir capital, equipamentos, tarefas, tempo, ou até mesmo largura de banda, devem ser bem alocados, nas quantidades corretas, nos momentos e durações corretas de forma a obter o melhor resultado possível. Normalmente os algoritmos de otimização são aplicados a problemas de difícil solução, e que envolvem significativas melhorias. Existem numerosas técnicas de otimização e a aplicação de cada uma delas depende essencialmente do tipo de problema (ILAB Sistemas, s.d.).

Tabela 3 - Tarifação energética

\begin{tabular}{lcc}
\hline Demanda $\left(\$ \mathrm{kWh}^{-1}\right)$ & HP & 26,44 \\
\hline & HFP & 7 \\
& Ultrap. HP & 52,88 \\
& Ultrap. HFP & 14 \\
Consumo $\left(\$ \mathrm{kWh}^{-1}\right)$ & HP Seco & 0,27873 \\
& HP Umido & 0,27873 \\
& HFP Seco & 0,18052 \\
& HFP Umido & 0,18052 \\
\hline
\end{tabular}

Programação por Restrições é um paradigma de programação que usa restrições para estabelecer as relações entre as variáveis. Na programação linear, as restrições usam as propriedades da solução a ser encontrada, sendo que uma restrição sobre uma sequência de variáveis é a relação entre essas restrições e seus domínios. As restrições podem serem vistas como um requisito que diz quais combinações de valores dos domínios das variáveis serão admitidas (Iizuka, 2012).

O conjunto de todos os possíveis estados do problema, onde se encontra a solução, denomina-se espaço de busca. Nas restrições, são formuladas condições que diminuem substancialmente a quantidade de buscas necessárias para se encontrar a solução do problema. Em tese, quanto maior for o número de restrições informadas ao sistema, menor será o número de possíveis soluções a serem analisadas e, portanto, melhor será o desempenho da aplicação (ILAB Sistemas, s.d.).

Neste estudo foram realizadas quatro simulações de sistemas de geração de energia elétrica a partir de biogás. Esses sistemas se baseiam na possibilidade de geração de energia dos geradores comerciais. Existem uma infinidade de grupos geradores de energia elétrica disponíveis no mercado. Os geradores utilizados estão descritos na Tabela 4.

Como os geradores são a diesel e são adaptados para que funcionem com biogás, o seu rendimento cai. Esse fato se deve ao menor rendimento do biogás em relação ao óleo diesel.

De acordo com Coldebella et al. (2008), a utilização do biogás como recurso energético se deve ao conteúdo de metano $\left(\mathrm{CH}_{4}\right)$ em sua composição. Em condições normais de temperatura e pressão (PTN) tem um poder calorífico inferior (PCI) de 9,9 $\mathrm{kWh} \mathrm{m}^{-3}$. A equivalência média do gás em relação ao diesel é de 0,56325, e o consumo médio por um motor de combustão interna é de $0,45 \mathrm{~m}^{3} \mathrm{HP}^{-1} \mathrm{~h}^{-1}$.

Com base na capacidade de geração dos gruposgeradores de energia foram calculadas as necessidades de produção de biogás, o tamanho de rebanho e volume do biodigestor. Além desses dados foram calculados também a o volume de produção de resíduos, necessidade energética, o e investimento necessário para a implementação do biodigestor e do grupo-gerador.

O biodigestor utilizado para este estudo, assim como por Noronha \& Gimenes (2008), é o com gasômetro 
de PVC, modelo Sansuy, cujas especificações técnicas foram dadas pela EMBRAPA Suínos e Aves, tendo como preço de referência $\mathrm{R} \$ 150,00$ da câmara de digestão.

Como método de otimização foi utilizado o método de Programação por Restrições explicado no item Método de Otimização.

Para este estudo utilizou-se os dados de consumo específico de Noronha \& Gimenes (2008) e de distribuição de gasto com energia de Ferrarez et al. (2010), como base de cálculo para o consumo energético da granja.

O objetivo do modelo foi de minimizar os custos envolvidos na implementação desse sistema e encontrar o número mínimo para o rebanho em cada caso. O software utilizado na otimização do problema foi o Lingo 14.0 Versão Demo. O Lingo é um software para otimização de problemas lineares e não lineares, programação linear entre outros problemas da pesquisa operacional. Este software permite utilizar uma sintaxe específica para modelagem de problemas de forma concisa e simples. A função objetivo da modelagem é representada por:

\section{Equação 3 - Função Objetivo}

Minimizar: $\mathrm{C} 1+\mathrm{C} 2+\mathrm{C} 3-\mathrm{C} 4$;

C1 - custo mensal de capital de geração de energia

C2 - custo do grupo-gerador

C3 - custo do biodigestor

C4 - receita mensal da venda de energia

As restrições foram elaboradas em relação as características levantadas com base na literatura. As restrições abordaram características como capacidade de produção de biogás, rendimento dos gruposgeradores, consumo dos grupos geradores, sua capacidade de geração de energia e seus custos.

\section{RESULTADOS E DISCUSSÃO}

Os resultados obtidos pelas simulações quando comparados a casos reais se mostram bastante razoáveis.

Porém alguns valores se mostram incompatíveis para uma análise válida. Um desses valores é o de necessidade energética de uma granja de suínos.

\section{Equação 4 - Resultados da simulação}

\begin{tabular}{lcccc}
\hline & Caso 1 & Caso 2 & Caso 3 & Caso 4 \\
\cline { 2 - 5 } Potência gerada (Wh) & 35.000 & 150.000 & 275.000 & 590.000 \\
\hline
\end{tabular}

Os valores de potência necessária para cada empreendimento apesar de terem sido estimados com base na literatura se mostraram a quem da necessidade real. Essa demanda na maioria das vezes varia de acordo com as instalações, as características construtivas, e características de manejo e gerenciamento de energia. Outro dado que necessita de um melhor levantamento de informações para sua especificação é o valor de implantação de biodigestores. Realizar cálculos baseados em estimativas é uma alternativa para métodos de otimização, porém quanto mais reais as informações mais factíveis são os resultados da otimização.

Apesar disso os dados de tamanho de rebanho se mostraram razoáveis e factíveis se comparados a empreendimentos reais. Nota-se que houve em todos os casos uma proporcionalidade entre os valores. Isso demonstra a validade dos resultados, de forma a manter o padrão de comportamento de crescimento dos valores em relação ao comportamento de crescimento do valor de investimento.

\section{CONCLUSÃO}

O modelo se mostrou útil para realização de estimativas de tamanho de rebanho necessário para a implantação de geradores de energia elétrica em granjas alimentados a partir de biogás.

Os valores levantados para a realização da modelagem se mostraram razoáveis, porém há a necessidade de mais pesquisas em relação aos valores de custo de implantação de biodigestores e em relação aos valores de necessidades energéticas da granja. Esses valores além de possibilitarem a elaboração de um modelo mais real possibilita também a realização de análises de viabilidade e planejamento econômico.

O modelo mostrou-se bastante interessante para estudos de caso, visto que para este tipo de pesquisa os valores mensurados em cada caso podem ser aplicados ao modelo obtendo assim soluções bastante precisas.

\section{LITERATURA CITADA}

Alves, J. W. et al., 2001. Nota técnica vii: Geração de energia a partir do biogás gerado por resíduos urbanos e rurais, São Paulo: Centro Nacional de Referência em Biomassa. 
Anon., s.d. Grupo Gerador de Energia á Diesel 150 Kva GBW150C-PRAMAC. [Online]. Available at: http://www.torchtools.com.br/loja/ produto.php?loja $=104633 \&$ IdProd=3405\&parceiro=3149. [Acesso em 1012 2013].

Anon., s.d. Grupo Gerador de Energia á Diesel 275 Kva GSW275V-PRAMAC. [Online] Available at: http://www.torchtools.com.br/loja/ produto.php?loja=104633\&IdProd=3409\&parceiro=3149. [Acesso em 1312 2013].

Anon., s.d. Grupo Gerador de Energia á Diesel 590 Kva GSW590V- PRAMAC. [Online] Available at: http: //www.torchtools.com.br/loja/ produto.php?loja $=104633$

$\&$ IdProd $=3417 \&$ parceiro=3149. [Acesso em 1012 2013].

Anon., s.d. Grupo Gerador Geramac S35-CL à Diesel | 35KVA - Trifásico 220V. [Online] Available at: http:// curitibamaquinasepecas.com.br/Produto-1GERADORES-DE-ENERGIA-Grupo-GeradorGeramac-S35-CL-a-Diesel-35KVA-Trifasico220V-versao-257-287.aspx. [Acesso em 1012 2013].

Coldebella, A., Souza, S. N. M. d., Ferri, P. \& Kolling, E. M., 2008. Geração de energia elétrica através de um motor gerador utilizando biogás da suinocultura. Paranaguá: s.n.

Embrapa Emater/RS Suinos e Aves., 2002. Coletânea de tecnologias sobre dejetos suínos. . Porto Alegre: Boletim Informativo BIPERS.

EMBRAPA, 2006. Boas Práticas de Produção de Suínos, Concórdia: EMBRAPA.

Ferrarez, A. H., Filho, D. O. \& Teixeira, C. A., 2010. INDEPENDÊNCIA ENERGÉTICA DE GRANJA SUINÍCOLA A PARTIR DO USO DE $B I O G A ́ S$, Viçosa: Engenharia na agricultura.

Godoy Júnior, E., Carrocci, L. R. \& Silveira, J. L., s.d. Biodigestores associados a sistema de cogeração para o aproveitamento do biogás produzido a partir de resíduos de suinocultura.. Guaratinguetá: UNIVERSIDADE ESTADUAL PAULISTA - UNESP Campus de Guaratinguetá Departamento de Energia.
Iizuka, V. d. A., 2012. Programação por Restrições aplicada a Problemas de Rearranjo de Genomas. Campinas: Universidade Estadual de Campinas.ILAB Sistemas, s.d. Tecnicas de Otimização. [Online]. Available at: http:// www.ilab.com.br/tecnicas.html. [Acesso em 07 12 2013].

Miele, M. \& Machado, J. S., 2010. Panorama da carne suína brasileira. [Online]. Available at: http://www.agroanalysis.com.br/ especiais_detalhe.php?idEspecial=54

Namuli, R., Pillay, P., Jaumard, B. \& Laflammec, C., 2013. Threshold herd size for commercial viability of biomass waste to energy conversion systems on rural farms. Quebec: Applied Energy.

Noronha, A. C. G. d. \& Gimenes, R. M. T., 2008. MENSURAÇÃO DOS CUSTOS DE IMPLANTAÇÃO DE BIODIGESTORES NA SUNINOCULTURA. Ribeirão Preto: V Encontro de Pesquisadores Latino-americanos de Cooperativismo.

Refosco, D., 2011. UTILIZAÇÃO DE RESÍDUOS DA SUINOCULTURA PARA PRODUÇÃO DE ENERGIA ATRAVÉS DO BIOGÁS E FERTILIZANTES ORGÂNICOS ESTUDO DE CASO: GRANJA MARMENTINI - DOIS VIZINHOS - PR, Curitiba: INSTITUTO DE ENGENHARIA DO PARANÁ - IEP.

Secretaria de Estado da Agricultura e do Aba Departamento de Economia Rural, 2013. Suinocultura - Análise da Conjuntura Agropecuária, s.1.: s.n.

Sindigás, s.d. Sindigás - Gás LP, Energia Brasileira. [Online]. Available at: http:// www.sindigas.com.br/Estatistica/ Default .aspx $?$ cat $=5 \&$ itemCount $=1$. [Acesso em 0912 2013].

Souza, S. N. M. d., Pereira, W. C. \& Pavan, A. A., s.d. Custo da eletricidade gerada em conjunto motor gerador utilizando biogás da suinocultura. Cascavel: UNIOESTE-CCET. 
Talamini, D. J. D., Martins, F. M., Arboit, C. \& Wolozsim, N., 2006. Custos agregados da produção integrada de suínos nas fases de leitões e de terminação., Concórdia: Custos e @ gronegócio on line.
Talamini, D. J. D., Martins, F. M., Arboit, C. \& Wolozsim, N., 2006. Custos agregados da produção integrada de suínos nas fases de leitões e de terminação.. s.1.:Custos e @ gronegócio on line.

Recebido para publicação em 31/11/2014 e aprovado em 19/01/2016. 